\title{
THE X-RAY BACKGROUND FROM STAR-FORMING GALAXIES
}

\author{
Richard E. Griffiths \\ Space Telescope Science Institute \\ 3700 San Martin Drive \\ Baltimore, MD 21218 USA
}

It has been established observationally that at least $30 \%$ of the all-sky X-ray background (XRB) in the energy range of 1-3 keV comes from active galactic nuclei (AGN) in the redshift interval of 0.4 to 1.2 (Griffiths et al. 1983, 1988); it is also quite plausible that AGN contribute half of the XRB in the 1-3 keV range (Morisawa and Takahara 1989). However, analysis of spatial fluctuations in the Einstein deep survey counts (Hamilton and Helfand 1987; Barcons and Fabian 1989) has indicated the presence of a relatively smooth component of the XRB with a corresponding surface density of discrete sources of at least several thousand per square degree.

Besides the difficulty of accounting for the smoothness of the XRB on both small and large scales, the greatest problem with the AGN interpretation of the whole of the XRB is the discrepancy between the hard XRB spectrum and the softer spectra of nearby quasars and Seyfert galaxies. We consider therefore an alternative or additional source for the all-sky $\mathrm{XRB}$, especially in the 3-20 keV energy range above that measured by the Einstein Observatory, viz. the integrated X-ray emission from an early population of low-metallicity massive $\mathrm{X}$-ray binaries (MXRBs) in regions of star formation. Such sources are typified by SMC X-1, with less luminous examples in our own Galaxy. This possibility was first mentioned by Bookbinder et al. (1979).

Population I X-ray binaries start emitting X-rays about $10^{7}$ years after the formation of the initial massive binary, and the epoch of X-ray emission probably lasts for at least $10^{5}$ to $10^{6}$ years. The presently active and known MXRBs in our Galaxy number about 20, with $\mathrm{X}$-ray luminosities of $\sim 10^{38} \mathrm{ergs} \mathrm{s}^{-1}$ and typical X-ray temperatures of $>15 \mathrm{keV}$.

Our Galaxy does not contain any low-metallicity MXRB, so sources like Cyg X-1 and Cen X-3 are low-luminosity counterparts to those found in star-forming galaxies. The nearest relevant examples are found in the Local Group, where the low metallicity of the SMC has been taken as the reason for the extraordinary X-ray output, rivaling the total $\mathrm{x}$-ray output of the Galaxy with only one-tenth the mass (Clark et al. 1978). The high-energy tail of binaries like SMC X-1 may be especially important in considerations of the XRB. Nearby galaxies containing superluminous sources include M82, M101, and possibly NGC 253. Long and van Speybroeck (1983) drew attention to the compact binaries in "normal" galaxies, where it is not unusual to find individual sources with luminosities in excess of $10^{39} \mathrm{ergs} \mathrm{s}^{-1}$ : M101 has three such sources, M100 has a source emitting in excess of $10^{40}$, and M82 has a source in an outlying arm with $L_{x}>10^{40} \mathrm{ergs} \mathrm{s}^{-1}$.

Ellis (1987) and others have observed excess blue emission in field galaxies at redshifts as low as $\mathbf{0 . 4}$, indicating the evolution of star formation in galaxies at very moderate redshifts. The number of MXRBs in these galaxies is expected to follow the size of the $\mathrm{H}$ II regions, or the number of $O$ stars. Such blue galaxies may be expected to have integrated X-ray emission of about $10^{41-42} \mathrm{ergs} \mathrm{s}^{-1}$ and to number perhaps $20-30 \%$ of all galaxies at those redshifts. 
One way of estimating the MXRB contribution is to use the luminosity function (LF) of line-emitting galaxies from the Center for Astrophysics redshift survey (Burg 1987) and to make an estimate based on the observed X-ray-to-optical luminosity ratio of emission-line galaxies. A few examples of relevant objects are contained, in fact, in the Einstein deep surveys. Objects in U Min and in Pavo at $z \approx 0.4$ may be representative of this class. The result of folding Burg's LF with the observed ratio of $l_{x} / l_{\text {opt }}$ is that the contribution to the XRB is $-30 \%$ at $2 \mathrm{keV}$. With hard $\mathrm{X}$-ray spectra, this fraction would be maintained or exceeded in the 3-20 keV range.

Young, Kleinmann, and Allen (1988) have found evidence that infrared (IR) starburst galaxies are powered by massive young stars in heavily obscured $\mathrm{H}$ II regions. Weedman (1986) constructed a luminosity function for starburst galaxies based on the $60 \mu \mathrm{m} I R A S$ counts and then used a ratio of IR-to-X-ray luminosity from a dozen objects that were observed both with IRAS and Einstein. This ratio was found to be $2.7 \times 10^{-8}$ from a sample chosen for their properties of unusual features generally arising from interactions (abnormality). Without invoking any evolution of starburst galaxies, an XRB contribution of at least $13 \%$ was found at $2 \mathrm{keV}$. The evolution of starburst galaxies has been discussed by Hacking, Condon, and Houck (1987); their "collision" models and "radio" models (especially the latter) would produce a contribution to the XRB dominating any other components.

Star-forming galaxies may contribute substantially - at least $\sim 20 \%$ and plausibly $\sim 50 \%$ - to the XRB in the 3-20 keV range. The first examples of these sources may already have been found in the Einstein deep surveys. The dominant contribution must arise from relatively low redshifts, i.e., at $z<1$, depending on the high-energy tail of the MXRB.

One prediction of the starburst origin of the XRB is, of course, that the starburst galaxies have hard X-ray spectra similar to those of MXRBs in the Local Group. Verification of this prediction will have to await $A X A F, X M M$, and other missions reaching $\mathrm{X}$-ray energies of 8-10 keV. This hypothesis would also predict a possible correlation between the fluctuations in the Einstein deep survey counts and deep radio source counts as well as for the data from the ROSAT deep surveys, which should be able to detect the soft X-ray components of the low redshift examples of these objects.

\section{REFERENCES}

Barcons, X. and Fabian, A. C. 1989, MNRAS, 237, 119.

Bookbinder, J. et al. 1979. Ap. J., 237, 647.

Burg, R. 1987, Ph. D. Thesis, MIT.

Clark, G. et al. 1978, Ap. J., 221, L37.

Ellis, R. 1987, in "Observational Cosmology," Proc. LAU Symposium No. 124, eds. G. Burbidge and L. Z. Fang, p. 367.

Griffiths, R. E. et al. 1983, Ap. J., 269, 375.

Griffiths, R. E. et al. 1988, in "The Post-Recombination Universe," NATO ASI Series C, eds. N. Kaiser and A. N. Lasenby, vol. 240, p. 91.

Hacking, P., Condon, J. J., and Houck, J. R. 1987, Ap. J., 316, L15.

Hamilton, T. T. and Helfand, D. J. 1987, Ap. J., 318, 93.

Long, K. S. and van Speybroeck, L. P. 1983, in "Accretion-driven Stellar X-ray Sources," eds. W. H. G. Lewin and E. P. J. van den Heuvel, p. 117.

Morisawa, K. and Takahara, F. 1989, PASJ, in press.

Weedman, D. W. 1986, in "Star Formation in Galaxies," NASA CP-2466, ed. Carol J. Lonsdale, p. 351.

Young, J. S., Kleinmann, S. G., and Allen, L. E. 1988, Ap. J., 334, L63. 